\title{
DESIGN AND CONSTRUCTION OF A BLUETOOTH CONTROLLED OBSTACLE AVOIDING VEHICLE
}

\author{
Konstantinos Charisis \\ Hellenic Naval Academy \\ Piraeus, Athens, Greece
}

\author{
Vyron Sokratous \\ Hellenic Naval Academy \\ Piraeus, Athens, Greece
}

\author{
Andreas Tsigopoulos \\ Hellenic Naval Academy \\ Piraeus, Athens, Greece
}

\author{
Argyris Stassinakis \\ National and Kapodistrian \\ University of Athens, Greece
}

\begin{abstract}
Aim of this project is to design a wireless controlled vehicle using Arduino, capable of avoiding obstacles and detecting motion when idle. The wireless control for this project is based on the Bluetooth protocol [1]. The construction of the vehicle has been implemented using an Arduino micro-controller, motor driver, ultrasonic sensor, Bluetooth module, PIR-based motion detection sensors and a buzzer. This paper presents the analysis of the design and construction of a vehicle with regards to hardware, software capable of movement control, obstacle detection, obstacle avoidance and motion detection. The vehicle constructed in its final form will be able to be remotely controlled, avoid obstacles and detect motion when in idling status. Initially the code will be simulated in software and then interfaced with the hardware. All the controls of the vehicle will be based on program embedded into Arduino.
\end{abstract}

Keywords - Remote Control Vehicle, Bluetooth Control, Motion Detection, Arduino, Collision Avoidance

\section{INTRODUCTION}

The research and development of autonomous vehicles dates back since the dawn of the twentieth century. There has been a great need for developing wirelessly remote-controlled unmanned vehicles for various purposes such as remote sensing of hostile environment and surveillance of unapproachable, or extremely difficult to access, territories. These vehicles can be integrated with cameras and sensors capable of detecting motion, measuring temperature and pressure of the atmosphere, analyzing the consistency of the air, identifying presence of humans, geopositioning and more [2-4]. Unmanned vehicles with such capabilities have many applications such as assisting humanity in cases of natural disasters, studying unexplored areas for scientific purposes and in military field. In this article a remotely controlled car has been designed and constructed and its operation is presented in terms of obstacle detection and avoidance using ultrasonic sensors. Furthermore, the vehicle includes a buzzer that warns the user when the distance between it and an obstacle has reached the set limit, as well as when a motion is detected while the scanning of motion is activated. The construction also includes LED indicators on the front and back side that turn on depending on the movement [5-7].

The rest of the paper is organized as follows. The designing and making of the vehicle are presented in section II. Section III refers to the programming algorithms used. Experimental results will be discussed in section IV. Concluding remarks are given in section $\mathrm{V}$.

\section{DESIGN AND CONSTRUCTION}

For the construction of the vehicle we used a 4WD chassis, where the HC-SR04 sensor was mounted on the front side [8]. In this way the reflected back echo signal from an object is taken as input to the sensor to determine the distance from it. A mobile phone or tablet is linked wirelessly to the vehicle, via the bluetooth module $\mathrm{HC}-05$, to control the movement and the rest sensors of the vehicle through an application. The bluetooth module is attached to the Arduino UNO [9-10], where inputs from the user are processed and translated into movement for the vehicle through motor driver L298N [11].

The vehicle is powered by two $3.7 \mathrm{~V}$ batteries in series connection and a breadboard is used to allow all the wirings of the project. Finally the three PIR motion detectors HC-SR501 [12], are mounted on the front, the right and the left side of the vehicle, and are activated upon user request, when the car does not move. When the PIR sensors detect motion, they are triggered and the buzzer sounds. Fig. 1 shows the complete circuit diagram of the $\mathrm{RC}$ vehicle. 
International Journal of Engineering Applied Sciences and Technology, 2020

Vol. 5, Issue 6, ISSN No. 2455-2143, Pages 8-11

Published Online October 2020 in IJEAST (http://www.ijeast.com)

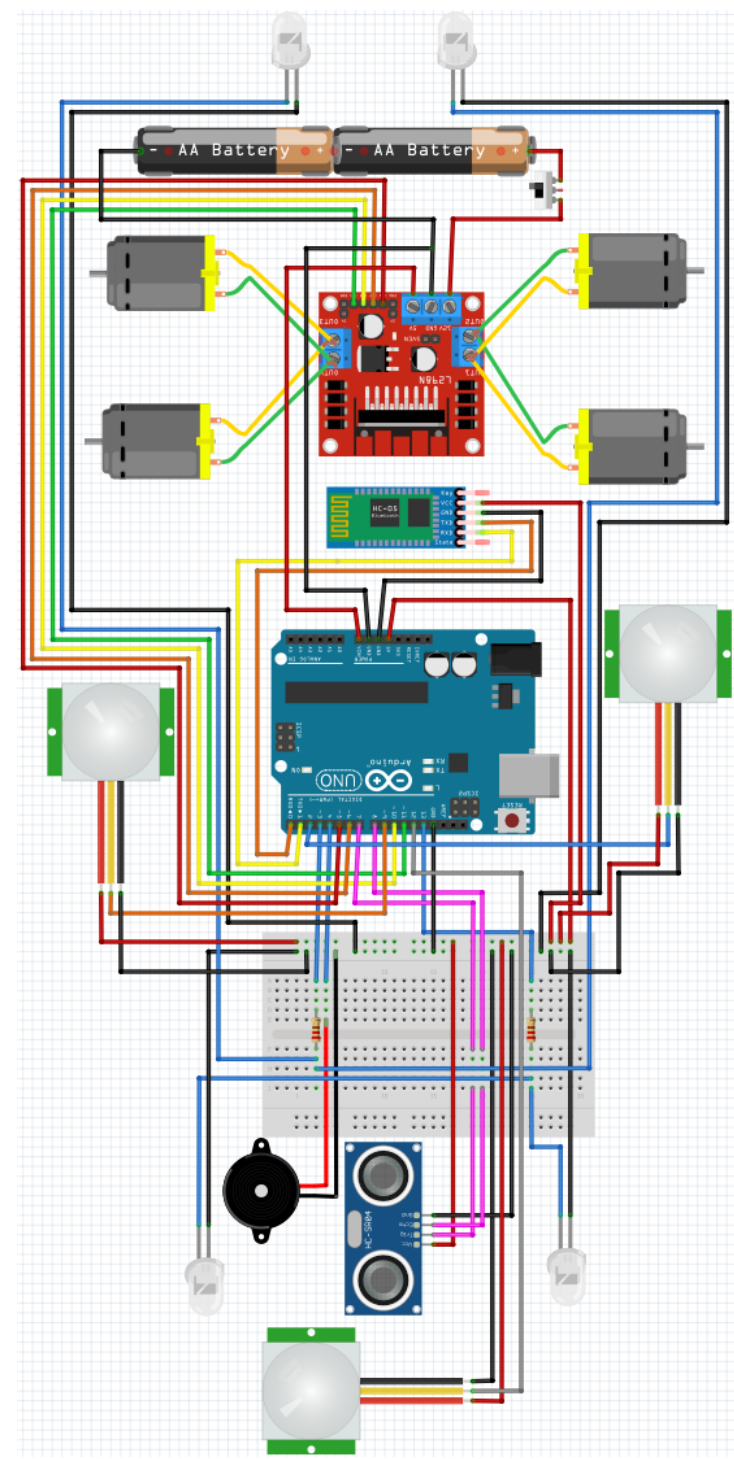

Fig. 1. Cicuit Diagram

\section{ALGORITHMS}

Firstly, we programmed the control keys so as to be able to move the car to all four directions (front, back, right and left). Secondly, we had to code the routine for the desired collision avoidance distance that would trigger the buzzer prompted by the ultrasonic sensor. This coding includes i) setting the distance, ii) stopping movement of the car in case the sensor measures distance less than the desired one and iii) reversing the vehicle to a safe distance. Moreover, when distance is measured less than the desired one by the ultrasonic sensor, a buzzer gives acoustic signal to warn the user.

For our project we mounted one sensor at the front side of the car and we set the safe distance to $30 \mathrm{~cm}$. In this case if the sensor detects an obstacle when moving forward and the measured distance is less than the set one, the sensor is triggered, the vehicle stops moving forward, the buzzer sounds a warning and backwards movement is initiated to the point that the obstacle is at least $30 \mathrm{~cm}$ away from it.

The final part of the coding is related with the PIR motion detection sensors. At this point we have to notice that if the sensors are activated while the vehicle moves, the PIR detectors will sense motion during its movement, and will be continuously triggered. This is not desirable, so for this project the coding allows the user to wirelessly activate the motion detectors upon request, when the car is not moving, so as to detect motion at a specific environment, sound the buzzer and inform the user when triggered.

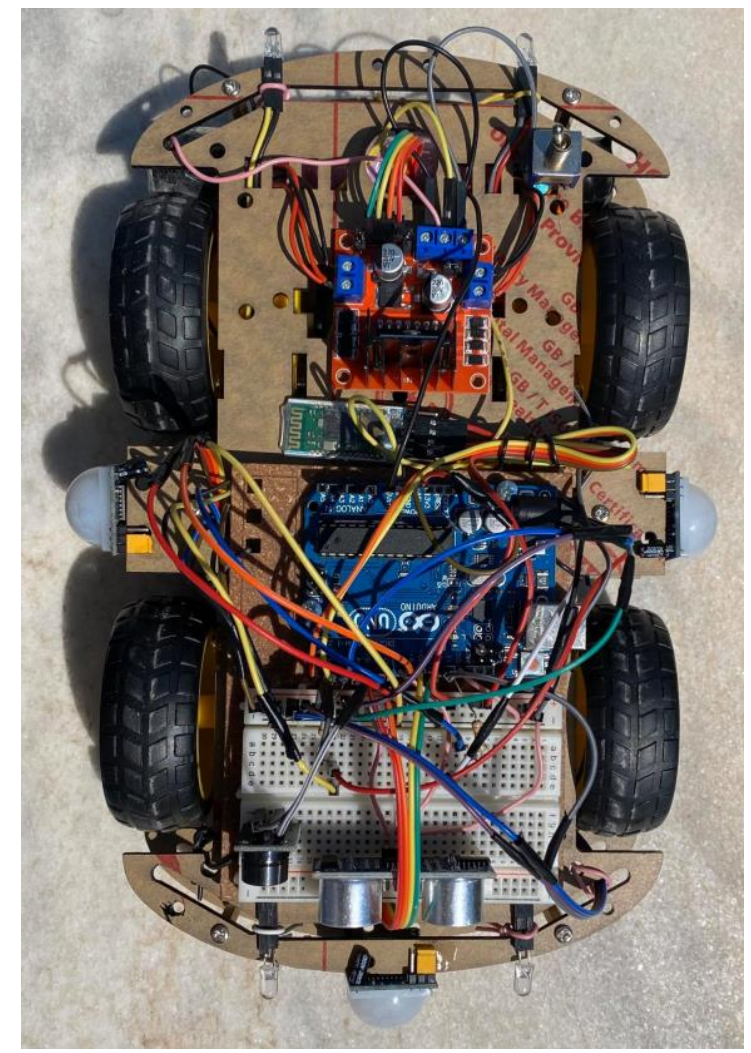

Fig. 2. Vehicle's electronic Circuit

\section{TESTING AND RESULTS}

We performed tests for every sensor individually so as to be certain that every part behaves accordingly. The next step was to integrate every component so as to construct the designed Bluetooth controlled obstacle avoiding vehicle. The final part of the testing was to fully test the vehicle in the real environment. The vehicle was controlled remotely by the user, it was able to move to any four directions and the avoid collision system would not let it hit any obstacle even when the user tried to drive the car towards it. The motion detection system could track every movement, when activated upon request, sound the buzzer and inform the user about motion in the nearby area. 


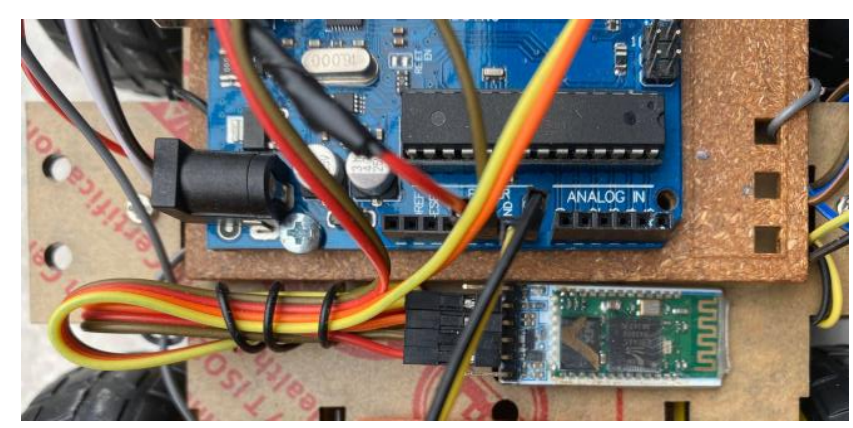

Fig. 3. Bluetooth module mounting and testing.
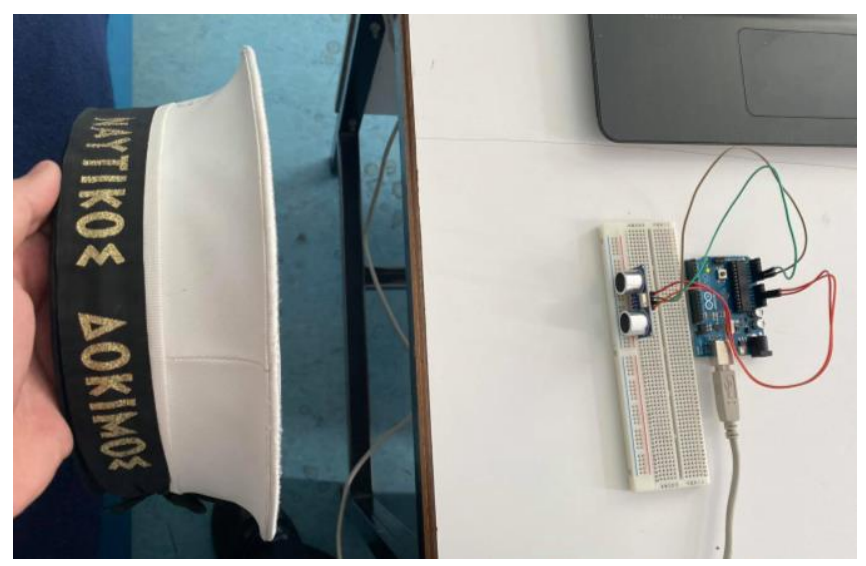

Fig. 4. Ultrasonic sensor testing.

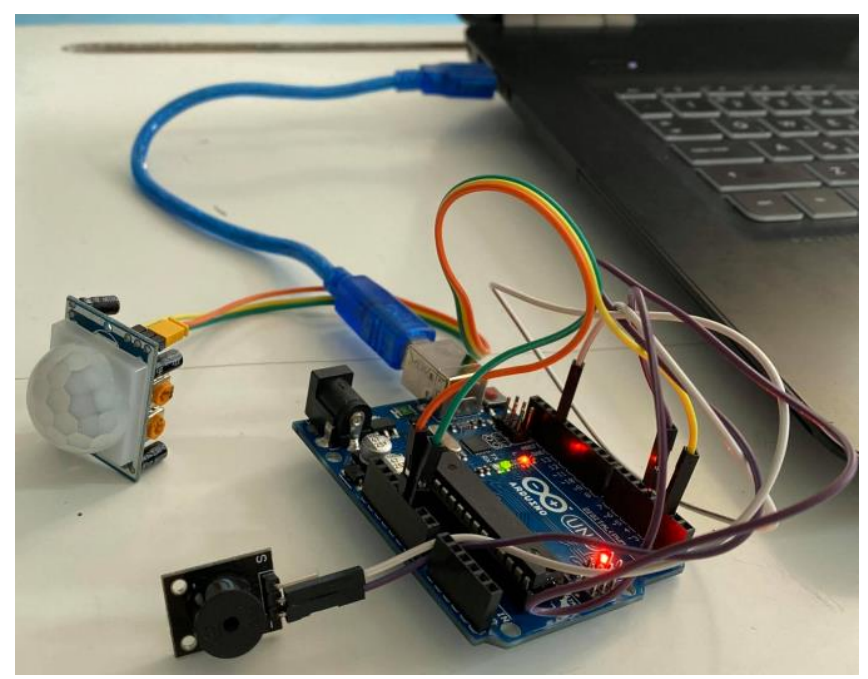

Fig. 5. PIR motion detection sensor and buzzer testing.

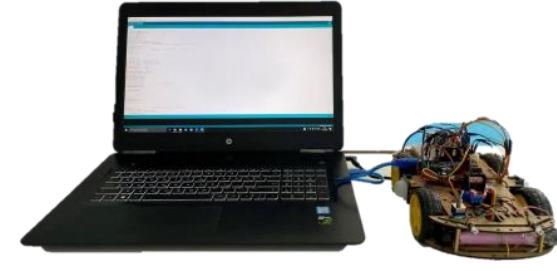

Fig. 6. Implementation of the code to the vehicle's Arduino processor.

\section{CONCLUSION}

We have presented the design and the construction of a wirelessly controlled obstacle avoiding vehicle with motion detection capabilities and demonstrated the satisfactory operation of it. Collision avoidance is important and can prevent accidents and even protect people during operations. There is always room for improvement and this applies on this project as well. Unmanned vehicles with the help of every type of sensors have unlimited capabilities and can assist humanity in every aspect.

\section{ACKOWLEDGMENT}

The authors acknowledge support from the Electronics Lab of the Hellenic Naval Academy.

\section{REFERENCES}

[1] Mohankumar, D. (2008). Bluetooth Technology. Retrieved May 27, 2020, from https://www.engineersgarage.com/contributions/blueto oth-technology/

[2] Gage, W. D. (1995). UGV History 101: A Brief History of Unmanned Ground Vehicle (UGV) Development Efforts. Unmanned Systems Magazine, vol. 13, No. 3, pp. 1-10.

[3] Galar, D., Kumar, U., Seneviratne, D. (2020). Robots, Drones, UAVs and UGVs for Operation and Maintenance. Doi.org/10.1201/9780429452260, ISBN: 9780429452260, CRC Press, Taylor \& Francis Group.

[4] Grabianowski, E. (2005). How Military Robots Work. Retrieved May 13, 2020, from: http://science.howstuffworks.com/military-robot2.htm/

[5] Williams, A. (2002). Microcontroller projects using the Basic Stamp. Doi.org/10.1201/9781482280777, ISBN: 9780429081309, CRC Press, Taylor \& Francis Group.

[6] De Simone, M., C., Guida, D. (2018). Identification and control of a Unmanned Ground Vehicle by using Arduino. UPB Scientific Bulletin, Series D: Mechanical Engineering, Vol. 80, Iss. 1, pp. 141-154.

[7] Bin Zabbar, MD., A., Chisty, N., A. (2016). Design \& Implementation of an Unmanned Ground Vehicle (UGV) Surveillance Robot. Int. Journal of Electrical 
and Electronics Engineering (IJEEE), Vol. 5, Iss. 6, Oct-Nov 2016, pp. 11-20.

[8] Ultrasonic Ranging Module HC - SR04. (n.d.). Retrieved May 27, 2020, from https://cdn.sparkfun.com/datasheets/Sensors/Proximity/ HCSR04.pdf

[9] Why Arduino? (2018). Retrieved May 27, 2020, from https://www.arduino.cc/en/Guide/Introduction

[10] Menhyárt, J., (2014). Concept of an UGV with Arduino Device. Military Engineer Journal, Issue: IX, vol. 2, pp. 140-148.

[11] Interface L298N DC Motor Driver Module with Arduino (2019). Retrieved May 27, 2020, from https://www.lastminuteengineers.com/1298n-dcstepper-driver-arduino-tutorial/amp/

[12]Dejan (2019). How PIR Sensor Works and How To Use It with Arduino. Retrieved May 27, 2020, from https://howtomechatronics.com/tutorials/arduino/howpir-sensor-works-and-how-to-use-it-with-arduino/ 